\title{
ANISOTROPIC QCD SUPERFLUIDS
}

\author{
JIŘÍ HOŠEK \\ Dept. Theoretical Physics, Nuclear Physics Institute \\ $25068 \check{R e z z}$ (Prague), Czech Republic
}

\begin{abstract}
We discuss two instances of anisotropic ordered quantum phases within QCD at finite baryon densities: (1) Fermionic deconfined three-color QCD matter with a spin one quark-quark Cooper pair condensate can exhibit distinct quantum i.e. low-temperature $(T)$ behaviors on macroscopic scales which bona fide can be observed in neutron stars. (2) Bosonic confined two-color QCD matter with a Bose-Einstein condensate of spin-one baryons can exhibit distinct quantum i.e. low- $T$ behaviors on macroscopic scales which can bona fide be observed in numerical lattice experiments.
\end{abstract}

\section{Introduction}

Understanding QCD starts with understanding its ground state: At zero baryon density $n_{B}$ (zero quark chemical potential $\mu$ ) and short distances the asymptotic freedom of QCD [1] translates into a weakly color-paramagnetic behavior of its perturbative vacuum [2]. Its excitations are the colored quarks, and the colored massless gluons. At zero baryon density and large distances the structure of QCD vacuum is a nightmare of particle physics. By expectation its excitations are the colorless hadrons, but their spectrum is not theoretically understood at present. Be it as it may, the only hadrons obliged to exist under a decent assumption on the QCD vacuum are the Nambu-Goldstone pions.

At nonzero quark chemical potential the problem of finding the ground state of QCD matter is apparently simpler: Very strong restriction on the QCD ground state i.e., its Lorentz invariance, is relaxed. The only property which remains sacred is the translation invariance. Price for relative simplicity of the ground state is the complexity of its excitations. They are of two types: (1) Quasiparticles are the excitations carrying the quantum numbers of the quantum fields defining the system. (2) Collective excita- 
tions are effectively described by composite operators constructed from the quantum fields. In both cases the lack of Lorentz invariance implies that the form of the dispersion law need not have the Einstein form. Quite often it can be reconstructed from thermodynamic behavior of the system.

Above the critical value $n_{B}^{c}$ of $n_{B}$ which marks the confinement-deconfinement phase transition quasiparticles of QCD matter are excited by the colored quark, and the colored-gluon quantum fields. Consequently, by definition for any number of colors $n_{c}$ the QCD matter at very low $T$ is a manybody system of interacting colored quarks and gluons. Its ground state is determined by the effective interactions relevant in the considered density range. Under rather general assumptions it is of the Cooper-pairing type. Non-relativistic condensed-matter relatives are the ordered many-fermion systems: the superconductors, the superfluid ${ }^{3} \mathrm{He}$ and, expectedly, the dilute quantum gases of fermionic atoms at extremely low $T$.

For $0<n_{B}<n_{B}^{c}$ all excitations of QCD matter are by definition collective and colorless. (1) For $n_{c}$ even (we will discuss explicitly only $n_{c}=2$ ) all hadrons are bosons. In particular, the ground-state baryons are bosons carrying the baryon number, and spin 0 or 1 . They can macroscopically occupy the ground state in any overall zero-momentum configuration. Non-relativistic condensed-matter relatives are the superfluid ${ }^{4} \mathrm{He}$, and the dilute gases of bosonic atoms. (2) For $n_{c}$ odd (we have in mind only the real world of $n_{c}=3$ ) the ground-state baryons are fermions carrying spin $1 / 2$ or $3 / 2$. Their ground state is most probably of the Cooper-pair condensate type.

At present, the "condensed-matter physics of QCD" [3] is quite popular. Reasons are both intellectual and practical. In this talk we discuss briefly the low- $T$ properties of two distinct ordered phases of QCD matter characterized by spontaneous breakdown of rotational symmetry:

(1) The two-flavor deconfined fermionic quark matter with both spin-0 and spin-1 diquark condensates is discussed in Sect.2. Such a mixture of isotropic and anisotropic color-superconducting phases could manifest experimentally in the interiors of the neutron stars.

(2) The dilute confined bosonic $n_{c}=2$ QCD matter of one massive flavor with Bose-Einstein condensate of spin-1 baryons is discussed in Sect.3. Properties of such matter exhibiting eventually superfluidity could be tested in numerical lattice QCD experiments. It is interesting though not surprising that the phenomenological description of soft Nambu-Goldstone modes is the same for both cases. 


\section{Anisotropic $n_{c}=3 \mathrm{QCD}$ matter of colored quarks}

There are good reasons to expect that the dynamics of the low- $T$ moderately dense deconfined $n_{c}=3$ colored-quark matter of two light flavors is governed by an effective Lagrangian of the $S U(3)_{c} \times S U(2)_{F} \times U(1)_{V} \times O(3)$ invariant form (detailed review of the subject with representative references is Michael Buballa's contribution [4]

$$
\mathcal{L}_{\text {eff }}=\bar{\psi}\left[i \not \partial-m+\mu \gamma_{0}\right) \psi+\mathcal{L}_{\text {int }}
$$

where $\mathcal{L}_{\text {int }}$ is a local four-fermion interaction to be determined experimentally. We make here a commonly accepted assumption that $\mathcal{L}_{\text {int }}$ is attractive in the quark-quark color anti-triplet antisymmetric channel. According to the Cooper theorem the system spontaneously reorganizes its Fermi sea into an energetically more favorable state characterized by the groundstate BCS-type quark-quark condensates. In accordance with Pauli principle their explicit form depends solely upon the details of $\mathcal{L}_{\text {int }}$.

One condensate

$$
\delta=\left\langle\psi^{T} C \gamma_{5} \tau_{2} \lambda_{2} \psi\right\rangle
$$

corresponding in an effective Ginzburg-Landau description to the groundstate expectation value of a complex spin-0 isospin- 0 color anti-triplet order parameter $\phi$ is "mandatory".

Since in (2) only the quarks of colors 1 and 2 participate we assume, following the suggestion of the pioneering Ref.[5] that the quark of color 3 undergoes the Cooper pairing in spin one [6]:

$$
\delta^{\prime}=\left\langle\psi^{T} C \sigma^{03} \tau_{2} \hat{P}_{3}^{(c)} \psi\right\rangle
$$

In an effective Ginzburg-Landau description $\delta^{\prime}$ corresponds to the groundstate expectation value of a complex spin-1 order parameter $\phi^{0 n} \equiv \phi_{n}$, and exhibits spontaneous breakdown of the rotational symmetry of the system.

Fermionic excitations above the condensates (2), (3) and the chiralsymmetry breaking one $\langle\bar{\psi} \psi\rangle$ are of two types:

(i) For colors 1 and 2 the dispersion law of Bogolyubov-Valatin quasiquarks is isotropic:

$$
E_{1}^{ \pm}(\vec{p})=E_{2}^{ \pm}(\vec{p}) \equiv E^{ \pm}(\vec{p})=\sqrt{\left(\epsilon_{p} \pm \mu\right)^{2}+|\Delta|^{2}} .
$$

Here $\epsilon_{p}=\sqrt{\vec{p}^{2}+M^{2}}, \Delta$ is the energy gap determined self-consistently, and found to be of the order $\sim 100 \mathrm{MeV}$ in model calculations; $M$ is an effective quark mass related to $\langle\bar{\psi} \psi\rangle$.

(ii) For color 3 the dispersion law is

$$
E_{3}^{ \pm}(\vec{p})=\sqrt{\left(\sqrt{M_{e f f}^{2}+\vec{p}^{2}} \pm \mu_{e f f}^{2}\right)^{2}+\left|\Delta_{e f f}^{\prime}\right|^{2}},
$$


where $\mu_{e f f}^{2}=\mu^{2}+\left|\Delta^{\prime}\right|^{2} \sin ^{2} \theta, M_{e f f}=M \mu / \mu_{e f f}$, and

$$
\left|\Delta_{e f f}^{\prime}\right|^{2}=\left|\Delta^{\prime}\right|^{2}\left(\cos ^{2} \theta+\frac{M^{2}}{\mu_{e f f}^{2}} \sin ^{2} \theta\right) .
$$

Here $\cos \theta=p_{3} /|\vec{p}|$, and the expected spontaneous breakdown of the rotational invariance is manifest. It is also worth of writing down explicitly the peculiar form of the gap equation for $\Delta^{\prime}$

$$
\Delta^{\prime}=16 H_{t} \Delta^{\prime} \int \frac{d^{3} p}{(2 \pi)^{3}}\left\{\left(1-\frac{\vec{p}_{\perp}^{2}}{s}\right) \frac{1}{E_{3}^{-}} \tanh \frac{E_{3}^{-}}{2 T}+\left(1+\frac{\vec{p}_{\perp}^{2}}{s}\right) \frac{1}{E_{3}^{+}} \tanh \frac{E_{3}^{+}}{2 T}\right\},
$$

where $s=\mu_{\text {eff }}\left(\vec{p}^{2}+M_{\text {eff }}^{2}\right)^{1 / 2}$. It is found by fixing the interaction (the coupling $\left.H_{t}\right)$, calculating the thermodynamic potential $\Omega(T, \mu)$, and imposing the condition $\partial \Omega / \partial \Delta^{\prime *}=0$.

Physical consequences of allowing for an anisotropic admixture in color superconductor are interesting: First, numerical analysis reveals extreme sensitivity of the anisotropic gap $\Delta^{\prime}$ on the chemical potential $\mu$, the details of interaction, and the cutoff $\Lambda$ needed to regularize the loop gap-equation integrals. Numerical values of $\Delta^{\prime}$ range from $\mathrm{O}(1 \mathrm{MeV})$ of early expectations [5] to the ones comparable with $\Delta$. Second, at very low temperatures the fermionic specific heat $c_{v}$ of the system is dominated by the quasiparticles of color $3[6]$ :

$$
c_{v} \approx \frac{12}{\pi} \frac{\mu^{2}+\left|\Delta^{\prime}\right|^{2}}{\left|\Delta^{\prime}\right|} T^{2}\left[1+\frac{\Delta_{0}^{\prime}}{T}+\frac{1}{2}\left(\frac{\Delta_{0}^{\prime}}{T}\right)^{2}+\frac{1}{6}\left(\frac{\Delta_{0}^{\prime}}{T}\right)^{3}\right] e^{-\frac{\Delta_{0}^{\prime}}{T}}
$$

It is interesting to notice that the low-lying quasiparticle spectrum around the minimum

$$
\Delta_{0}^{\prime}=\frac{M\left|\Delta^{\prime}\right|}{\sqrt{\mu^{2}+\left|\Delta^{\prime}\right|^{2}}} .
$$

takes the form

$$
E_{3}^{-}\left(p_{\perp}, p_{3}\right) \approx \sqrt{\Delta_{0}^{\prime 2}+v_{\perp}^{2}\left(p_{\perp}-p_{0}\right)^{2}+v_{3}^{2} p_{3}^{2}}
$$

i.e., $\Delta_{0}^{\prime}$ vanishes for $M=0$, and the specific heat becomes quadratic in $\mathrm{T}$ [7].

Third, because for $\Delta^{\prime}$ different from zero the condensate (3) breaks the $O(3) \times U(1)$ symmetry of the model spontaneously, the spectrum of the system should contain the collective Nambu-Goldstone (NG) modes. Due to the Lorentz-noninvariance of the model there can be subtleties $[8,9,10]$. The 
NG spectrum can be analyzed [6] within an underlying effective GinzburgLandau potential

$$
V(\phi)=-a^{2} \phi_{n}^{\dagger} \phi_{n}+\frac{1}{2} \lambda_{1}\left(\phi_{n}^{\dagger} \phi_{n}\right)^{2}+\frac{1}{2} \lambda_{2} \phi_{n}^{\dagger} \phi_{n}^{\dagger} \phi_{m} \phi_{m}
$$

for the complex order parameter $\phi_{n}$ [9], with $\lambda_{1}+\lambda_{2}>0$ for stability. For $\lambda_{2}<0$ the ground state is characterized by $\phi_{\text {vac }}^{(1)}=\left(\frac{a^{2}}{\lambda_{1}}\right)^{1 / 2}(0,0,1)$ which corresponds to our Ansatz Eq. (3) for the BCS-type diquark condensate $\delta^{\prime}$. This solution has the property $\langle\vec{S}\rangle^{2}=\left(\phi_{\text {vac }}^{(1) \dagger} \vec{S} \phi_{v a c}^{(1)}\right)^{2}=0$. The spectrum of small oscillations above $\phi_{v a c}^{(1)}$ consists of $1+2 \mathrm{NG}$ bosons, all with linear dispersion law: one zero-sound phonon and two spin waves [9]. Implying a finite Landau critical velocity, this fact is crucial for a macroscopic superfluid behavior of the system [10].

\section{Anisotropic $n_{c}=2$ QCD matter of colorless spin-1 baryons}

For understanding the confining QCD vacuum medium the number of colors $n_{c}$, once bigger than one, does not seem to be a crucial parameter of the QCD Lagrangian. Introducing the quark chemical potential into it changes, however, the situation dramatically: According to QCD dogmas for $n_{c}$ odd/even the colorless baryons are fermions/bosons. Consequently, the quantum i.e. low- $\mathrm{T}$ behavior of many-baryon systems must be markedly different in worlds with three and two colors. In particular, and most important, integer-spin many-baryon systems can exhibit under specific conditions (low densities, weak, repulsive two-body interactions) the Bose-Einstein condensation. This phenomenon certainly implies spontaneous breakdown of the global $U(1)$ symmetry generated by the operator of baryon charge, and in the case of spin-1 baryons also of the $O(3)$ rotational symmetry. Again, the system should contain phenomenologically important and theoretically interesting gapless NG modes.

The fact that the gedanken world of $n_{c}=2$ QCD matter at finite $\mu$ is, unlike the real $n_{c}=3$ one, accessible to the first-principle lattice computations is alluring: Lattice results can replace true experimental data, and provide in principle ideal tests of analytic models not only of the very hadron formation but also of the hadron-hadron interactions.

At present the majority of $n_{c}=2$ QCD studies at finite $\mu$ is devoted to the regime of approximate chiral symmetry. Assumption of its spontaneous symmetry breakdown is of course good : (1) It is natural because for $n_{c}=3$ and and the number of flavors $n_{F}=2,3$ it is an experimental fact. (2) It is interesting because (i) dealing with the symplectic group its pattern is generically different from the case of $n_{c}=3$; (ii) it may yield relativistic 
vector condensation [11].(3) It is predictive because the powerful chiral perturbation theory can be employed.

We believe it is both useful and interesting to study the $n_{c}=2$ low- $T$ integer- spin baryonic matter also in the regime of no chiral symmetry. We have in mind in particular the simple and conceptually clean case of one electrically neutral massive flavor: First, quark masses are the parameters external to QCD, and their ratio to $\Lambda_{Q C D}$, another theoretically arbitrary parameter of QCD, can be chosen at will. Second, an unknown confining dynamics is not masked by the chiral one. Third, according to the QCD dogmas there should exist just one type of the colorless massive spin-1 baryon described at small density characterized by $\mu$ by a non-relativistic second-quantized Schroedinger field $\phi_{n}$.

Employing with pleasure the principle of the least action we refer again to the papers [9] dealing with the description of Bose-Einstein condensation of dilute spin-1 gases: Their analysis applies here as it applied also to the formally identical Hamiltonian of Ginzburg-Landau of Sect.2. The $O(3) \times$ $U(1)$ invariant Hamiltonian density of a weakly-interacting Bose gas of Bogolyubov dealing with spin- 1 baryons $\phi_{n}$ of mass $m$ is:

$$
H=\phi_{n}^{\dagger}\left(-\frac{\nabla^{2}}{2 m}-\mu\right) \phi_{n}+\frac{1}{2} \lambda_{1}\left(\phi_{n}^{\dagger} \phi_{n}\right)^{2}+\frac{1}{2} \lambda_{2} \phi_{n}^{\dagger} \phi_{n}^{\dagger} \phi_{m} \phi_{m},
$$

In contrast to the many-fermion case of previous section we believe that the phenomenological baryon-baryon couplings $\lambda_{1}$ and $\lambda_{2}$ in (12) are calculable with reasonable accuracy both numerically on the lattice, and within models pretending to describe the confinement of color. The point is that only one of the two generically different patterns of the NG boson spectrum can exist [9] for given parameters of the primary QCD Lagrangian:

(i) For $\lambda_{1}>0, \lambda_{2}>0$ the homogeneous condensate

$$
\left\langle\Phi_{n}\right\rangle=\sqrt{\frac{n_{0}}{2}}(1, i, 0)
$$

describes a ferromagnetic configuration $\left(\mu=n_{0} \lambda_{1}\right)$. For very small momenta the spectrum of small oscillations above the condensate (13) contains soft NG modes having the dispersion law both linear, and quadratic in $|\vec{p}|$.

(ii) For $\lambda_{1}>0, \lambda_{2}<0$ the homogeneous condensate

$$
\left\langle\Phi_{n}\right\rangle=\sqrt{n_{0}}(1,0,0)
$$

describes a polar configuration $\left(\mu=n_{0}\left(\lambda_{1}+\lambda_{2}\right)\right)$. For very small momenta the spectrum of small oscillations above the condensate (14) contains soft NG modes all having linear dispersion laws. 


\section{Conclusion}

What we have presented in this contribution are two intuitively simple, hopefully interesting, and bona fide testable illustrations of richness of the condensed matter physics aspects of QCD- both under reasonable theoretical control. In particular, lack of Lorentz invariance due to the chemical potential implies interesting properties of the NG boson spectrum.

What we would have liked to present is less simple: We speculate that the finite- $\mu$ studies of 2-color QCD might be useful also to condensed-matter physics: Strongly interacting (i.e. dense) integer-spin baryon system can have a "superfluid" ground state without Bose-Einstein condensate, and hence be relevant for microscopic understanding of the superfluid ${ }^{4} \mathrm{He}$.

This work was supported in part by grant GACR 202/02/0847. I am grateful to Jiři Adam, Michael Buballa, Micaela Oertel and Adriano Di Giacomo for many pleasant discussions.

\section{References}

1. Gross, D.J., and Wilczek, F. (1973) Ultraviolet behavior of nonabelian gauge theories, Phys. Rev. Lett., 30, pp. 1343-1346;

Politzer, H.D.(1973) Reliable perturbative results for strong interactions ?, Phys. Rev. Lett., 30, pp. 1346-1349

2. Nielsen, N.K. (1981) Asymptotic freedom as a spin effect, Am. J. Phys., 49, pp. 1171-1178;

Hughes, R.J. (1981) More comments on asymptotic freedom, Nucl. Phys., B 186, pp. $376-412$

3. Rajagopal, K. and Wilczek, F. (2001) The condensed Matter Physics of QCD, in Shifman, M. (ed.), B.L. Ioffe Festschrift, At the Frontier of Particle Physics/ Handbook of QCD, vol.3, World Scientific, Singapore, pp.2061-2151

4. Buballa, M. (2002) Color superconductivity in two- and three-flavor systems at moderate densities, e-Print archive, hep-ph/0205027, these proceedings

5. Alford, M., Rajagopal, K., and Wilczek, F. (1998) QCD at Finite Baryon Density: Nucleon Droplets and Color Superconductivity, Phys. Lett., B 422, pp. 247-256

6. Buballa, M., Hošek, J., and Oertel, M. (2002) Anisotropic admixture in colorsuperconducting quark matter, e-Print archive, hep-ph/0204274

7. Leggett, A. J. (1975) A theoretical description of the new phases of liquid ${ }^{3} \mathrm{He}$, Rev. Mod. Phys., 47, pp. 331-414

8. Nielsen, H., and Chanda, S. (1976) On how to count Goldstone bosons, Nucl. Phys., B 105, pp. 445-463;

Leutwyler, H. (1994) Nonrelativistic effective Lagrangians, Phys. Rev., D 49, pp. 3033-3043;

Schafer, T., Son, D.T., Stephanov, M.A., Toublan, D., and Verbarschoot, J.J. (2001) Kaon condensation and Goldstone's theorem, Phys. Lett., B 522, pp. 67-75;

Sannino, F., and Schafer, W. (2002) Relativistic massive vector condensation, Phys. Lett., B 527, pp. 142-148

9. Ho, T.-L. (1998) Spinor Bose Condensates in Optical Traps, Phys. Rev. Lett., 81, pp. $742-745$;

Ohmi, T., and Machida, K. (1998) Bose-Einstein condensation with internal degrees of freedom in alkali atom gases, e-Print archive, cond-mat/9803160

10. Miransky, V.A., and Shovkovy, I.A. (2002) Spontaneous Symmetry Breaking with 
Abnormal Number of nambu-Goldstone Bosons and Kaon Condensate, Phys. Rev. Lett., 88, pp. 111601-4

11. Lenaghan, J.T., Sannino, F., and Splittorf, K. (2002) The superfluid and conformal phase transitions of two-color QCD, Phys.Rev., D 65, pp. 054002-16;

Sannino, F., and Schafer, W. (2002) Vector condensation at large chemical potential, e-Print archive, hep-ph/0204353 